\title{
Isolating Attosecond Electron Dynamics in Molecules where Nuclei Move Fast
}

\author{
Laura Cattaneo, ${ }^{1, * \dagger}$ Luca Pedrelliø, ${ }^{1, \dagger}$ Roger Y. Bello, ${ }^{2}$ Alicia Palacios, ${ }^{3,4}$ \\ Phillip D. Keathley $\odot,{ }^{5}$ Fernando Martín, ${ }^{3}$ and Ursula Keller ${ }^{1}$ \\ ${ }^{1}$ Physics Department, Institute of Quantum Electronics, ETH Zürich, 8093 Zürich, Switzerland \\ ${ }^{2}$ Chemical Sciences Division, Lawrence Berkeley National Laboratory, Berkeley California 94720, USA \\ ${ }^{3}$ Departamento de Química, Módulo 13, Universidad Autónoma de Madrid, 28049 Madrid, Spain \\ ${ }^{4}$ Institute for Advanced Research in Chemical Sciences (IAdChem), Universidad Autónoma de Madrid, 28049 Madrid, Spain \\ ${ }^{5}$ Research Laboratory of Electronics, Massachusetts Institute of Technology, Cambridge, Massachusetts 02139, USA
}

(Received 22 October 2021; revised 13 December 2021; accepted 13 January 2022; published 10 February 2022)

Capturing electronic dynamics in real time has been the ultimate goal of attosecond science since its beginning. While for atomic targets the existing measurement techniques have been thoroughly validated, in molecules there are open questions due to the inevitable copresence of moving nuclei, which are not always mere spectators of the phototriggered electron dynamics. Previous work has shown that not only can nuclear motion affect the way electrons move in a molecule, but it can also lead to contradictory interpretations depending on the chosen experimental approach. In this Letter we investigate how nuclear motion affects and eventually distorts the electronic dynamics measured by using two of the most popular attosecond techniques, reconstruction of attosecond beating by interference of two-photon transitions and attosecond streaking. Both methods are employed, in combination with ab initio theoretical calculations, to retrieve photoionization delays in the dissociative ionization of $\mathrm{H}_{2}, \mathrm{H}_{2} \rightarrow \mathrm{H}^{+}+\mathrm{H}+e^{-}$, in the region of the $Q_{1}$ series of autoionizing states, where nuclear motion plays a prominent role. We find that the experimental reconstruction of attosecond beating by interference of two-photon transitions results are very sensitive to bond softening around the $Q_{1}$ threshold $(27.8 \mathrm{eV})$, even at relatively low infrared (IR) intensity $\left(I_{0} \sim 1.4 \times 10^{11} \mathrm{~W} / \mathrm{cm}^{2}\right)$, due to the long duration of the probe pulse that is inherent to this technique. Streaking, on the other hand, seems to be a better choice to isolate attosecond electron dynamics, since shorter pulses can be used, thus reducing the role of bond softening. This conclusion is supported by very good agreement between our streaking measurements and the results of accurate theoretical calculations. Additionally, the streaking technique offers the necessary energy resolution to accurately retrieve the fastoscillating phase of the photoionization matrix elements, an essential requirement for extending this technique to even more complicated molecular targets.

DOI: 10.1103/PhysRevLett.128.063001

In the past 20 years, a wide range of experimental techniques have been developed to measure attosecond electron dynamics in real time [1-9]. One in particular has been extensively used because of its applicability for current experimental setups: the so called reconstruction of attosecond beating by interference of two-photon transitions (RABBITT). RABBITT is an interferometric technique that encodes time-delay information in the oscillating spectral phase of interfering photoemitted electron wave packets, which is sampled discretely over the energy bandwidth covered by an attosecond pulse train (APT). While the limitations of this discrete sampling can be

Published by the American Physical Society under the terms of the Creative Commons Attribution 4.0 International license. Further distribution of this work must maintain attribution to the author(s) and the published article's title, journal citation, and DOI. Open access publication funded by the Max Planck Society. overcome $[5,7,9]$ to provide complete time-delay information in simple atomic systems, when molecular targets are involved, the presence of nuclear degrees of freedom significantly increases the complexity or even renders RABBITT analysis impossible. For instance, to implement RABBITT-like techniques in the near-infrared (NIR), the probe pulse duration is on the order of $30 \mathrm{fs}$ or longer. Exposing molecular targets to such long NIR pulses can trigger nuclear dynamics that contaminate the electron dynamics of interest.

Another method that has been widely used to obtain photoionization time delays in atomic systems is attosecond streaking [4]. In this technique photoelectrons are generated by exposing the target atom or molecule to a single attosecond pulse (SAP) of extreme ultraviolet (XUV) light in the presence of a few-cycle NIR laser pulse. The photoionization time delay is encoded in the phase of the dipole transition matrix element shaping the obtained spectrogram. There are two notable aspects of attosecond streaking. First, 
it provides continuous phase spectra [10,11], leading to improved energy resolution in the extraction of time-delay information. Second, and more importantly for systems with fast moving nuclei like $\mathrm{H}_{2}$, streaking does not require the use of long NIR pulses. The electron dynamics can be retrieved from few-femtosecond-long regions in time just after photoionization. Despite these advantages experimental and theoretical work on molecular streaking is scarce as adequate retrieval algorithms required to extract the desired photoionization time delay information have only recently been developed [12-14].

In this Letter, we use a recently developed retrieval algorithm [15] to demonstrate the advantages of attosecond streaking for extracting temporal information of electron dynamics generated by XUV light on $\mathrm{H}_{2}$ in the energy region where doubly excided states (DESs) decay and dissociation of the remaining molecular cation is faster. $\mathrm{H}_{2}$ is the ideal benchmark, since very precise theoretical calculations can be performed taking into account all relevant electronic and nuclear degrees of freedom and the coupling between them. For a clear identification of the different effects, we consider a fixed molecular orientation, which requires combining the usual streaking and RABBITT setups with multicoincidence detection of electrons and ionic fragments. To ease the comparison with theory, we focus on $\mathrm{H}_{2}$ molecules parallel to the polarization direction of the ionizing radiation, which reduces the optically open ionization channels. We unambiguously show that delays obtained from RABBITT measurements are very sensitive to bond softening effects even at relatively low NIR intensity, while delays resulting from streaking measurements are rather insensitive to this effect, leading to phase-delay profiles that are in very good agreement with the theoretical calculations.

In our experiments, we photoionize a mixture of $\mathrm{Ne}$ (10\%) and randomly oriented $\mathrm{H}_{2}$ molecules (90\%) with either the XUV-SAP or APT having photon energy spectra ranging from 20 to $40 \mathrm{eV}$ (see Supplemental Material [16]). In this spectral region, $\mathrm{H}_{2}$ presents a multitude of states which contribute to the photoelectron spectrum resulting after one-photon absorption as shown in Fig. 1(a) and summarized in Table I. Different channels are accessible depending on the molecular orientation with respect to the light polarization ( $\|$ and $\perp$ ) and photon energies, leading to distinct kinetic energy release (KER, in formulas as $E_{k}$ ) $\left(\mathrm{H}^{+}+\mathrm{H}\right)$. Thus, retrieving the molecular orientation at the moment of ionization and the KER of the dissociated nuclei allow one to distinguish photoelectrons associated with a specific ionization channel [15].

We detect the fragment ions $\left(\mathrm{Ne}^{+}, \mathrm{H}_{2}^{+}\right.$, and $\left.\mathrm{H}^{+}\right)$in coincidence with the photoelectron generated in the same photoionization event by using a cold target recoil ion momentum spectroscopy (COLTRIMS) detector, in order to separate photoelectrons belonging to different gas species $\left(\mathrm{Ne}\right.$ and $\mathrm{H}_{2}$ ) and access the angular resolved photoionization dynamics $[17,18]$. In particular, for the dissociative ionization channel, we can access the molecular orientation $\beta$ of the molecule at the moment of ionization [see inset in Fig. 1(a)], assuming the validity of the axial recoil approximation [19-21], see Supplemental Material [16]. We further extract the spectral phase accumulated by the photoemitted electrons, exploiting the attosecond streaking and the RABBITT techniques, as shown in Figs. 1(b) and 1(c), respectively.
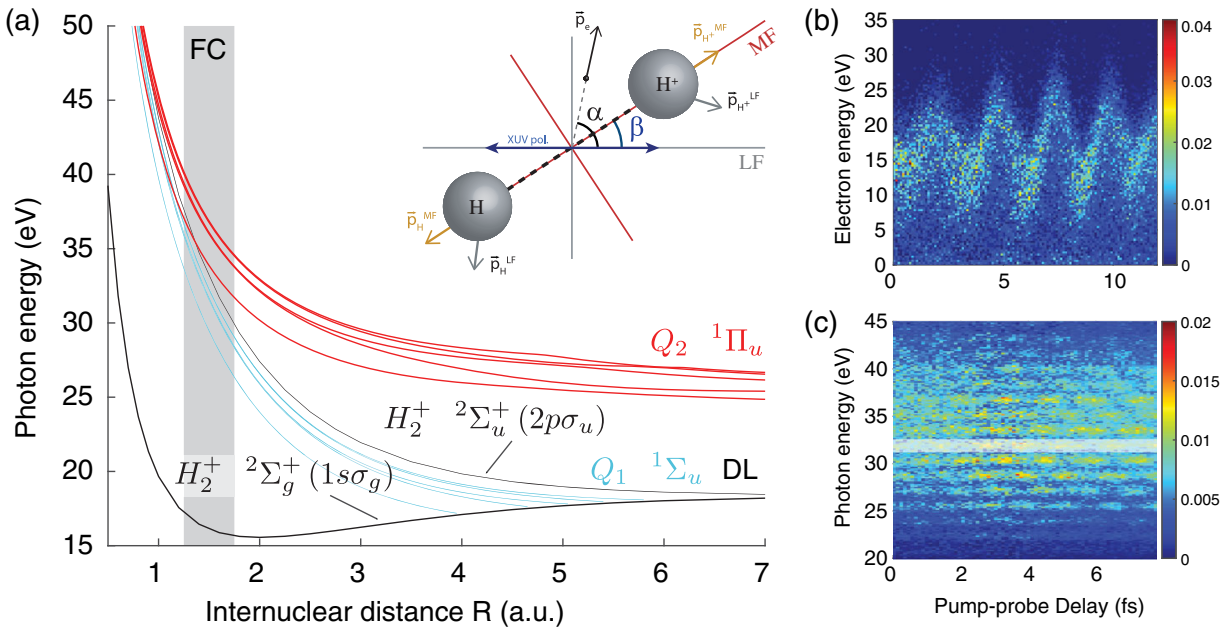

FIG. 1. (a) Potential energy surfaces involved in the ionization of $\mathrm{H}_{2}$. Direct photoionization pathways into the dissociative state are indicated by the black curves. Indirect pathways bring the molecule to the dissociation via autoionizing DESs of the first series $Q_{1}$ (blue curves) and of the second series $Q_{2}$ (red curves). The Franck-Condon (FC) region is indicated by the gray area around the equilibrium distance of $\mathrm{H}_{2}$ of $R_{\mathrm{eq}}=1.4$ a.u. The inset sketches the passage from laboratory (LF) to molecular frame (MF) and involved angles considered in this Letter $(\alpha$ and $\beta$ ). (b) and (c) represent examples of attosecond streaking and RABBIT experimental traces when selecting on the $H^{+}$fragments for $\beta=0 \pm 20^{\circ}$ and $\alpha=0 \pm 30^{\circ}$ with respect to the XUV polarization axis. 
TABLE I. Photoionization channels in $\mathrm{H}_{2}$ after absorption of photon energies in the range $20-40 \mathrm{eV}$. Specifically, the final state, the required XUV energy to populate each state, and the dominant $\mathrm{H}_{2}$ molecular orientation are listed.

\begin{tabular}{lcc}
\hline \hline Photoionization channel & $E_{\mathrm{XUV}}(\mathrm{eV})$ & Orientation \\
\hline Direct $1 s \sigma_{g}$ & Ionization $>15.44$ & $\|$ and $\perp$ \\
& Dissociative $>18.1$ & \\
Indirect $Q_{1} \rightarrow 1 s \sigma_{g}$ & $25-36$ & $\|$ \\
Direct $2 p \sigma_{u}$ & $\geq 30$ & and $\perp$ \\
Indirect $Q_{2} \rightarrow 1 s \sigma_{g}$ & $\geq 31$ & $\perp$ \\
Indirect $Q_{2} \rightarrow 2 p \sigma_{u}$ & $\geq 31$ & $\perp$ \\
\hline \hline
\end{tabular}

For the attosecond streaking we used a SAP with a center energy of $35 \mathrm{eV}$ and a bandwidth spanning from 25 to $41 \mathrm{eV}$, see Supplemental Material [16]. The resulting photoionized electron wave packet (EWP), as shown in Fig. 1(b), will present a broad energy distribution, allowing us to access the dipole phase information in a continuous manner over the entire SAP energy spectrum. The attosecond streaking is expected to be a more sensitive technique than RABBITT when a dense series of states are populated. This is often the case with molecular targets, or when sharp variations of the dipole phase are expected, for example due to the presence of autoionizing states.

By using this coincident detection, it is possible to disentangle the streaking spectrograms generated by electrons photoemitted from $\mathrm{Ne}^{+}$(see Supplemental Material Fig. S2.1(b) [16]), from $\mathrm{H}_{2}^{+}$(see Supplemental Material Fig. S2.1) and $\mathrm{H}^{+}$[Fig. 1(b)]. The $\mathrm{Ne}^{+}$spectrogram always plays the role of a reference. Ne has been chosen as reference gas for two reasons: it does not present atomic resonances in the chosen energy region $20-40 \mathrm{eV}$ and the $\mathrm{Ne}$ spectral phase contribution can be extracted from the argument of the complex dipole transition matrix element (DTME) available in literature [22].

In the streaking measurements, the EWP phase is not immediately accessible and a retrieval algorithm is required. Recently, Pedrelli et al. developed a new algorithm called ACDC that can extract the EWP phase information with no approximations beyond the strong field approximation [17]. The retrieval process has two steps. First we consider the reference streaking spectrogram target, Ne in our case, assuming a well-known DTME [22], to access to the XUV-SAP and NIR pulses used in the experiment [23]. Second, based on the retrieved XUV and NIR pulses, the ACDC retrieves the target spectrograms, in our case $\mathrm{H}_{2}^{+}$and $\mathrm{H}^{+}$, to access their unknown absolute DTMEs and corresponding spectral phase.

Multiple time windows ( $\sim 4 \mathrm{fs}$ ) of the pump-probe delay of the reference and target spectrogram have been considered ensuring a more accurate characterization of the XUV-SAP and IR pulse, due to possible pulse modifications over the course of the measurement. A weighted average of the ACDC reconstructions (considering the reconstruction error and the number of reconstructed phases) provides the mean and the standard deviation of the phase of the DTME term [24], see Supplemental Material [16].

RABBITT, on the other hand, is an attosecond interferometric technique that directly encodes the spectral phase, hence the time-delay information, in the so-called sideband (SB) signal [see Fig. 1(b), white rectangle]. In each SB, two different two-photon absorption processes can contribute, and therefore, interfere, capturing the relative phase between these quantum paths. Since the APT can be expressed, in the spectral domain, as a comb of high-order harmonics (HHs) spaced by twice the IR driving field frequency $2 \omega_{\mathrm{IR}}$, absorption of one $\mathrm{HH}_{2 q+1}$ and the stimulated emission of one NIR photon. Such a $S_{2 q}$ signal oscillates as a function of the pump-probe delay $\tau$ due to the quantum-path interference:

$$
S_{2 q}(\tau) \propto \cos \left(2 \omega_{\mathrm{IR}} \tau-\varphi\right) .
$$

The extracted phase shift $\varphi$ contains contributions from the scattering phase acquired by the photoelectron as it recedes in the molecular potential, and two measurementinduced terms: the XUV-chirp and the continuumcontinuum phases. The well-known Wigner time delay $\tau_{W}$ is given by the energy derivative of the scattering phase [25].

While the absolute ionization time, and thus absolute phase term, is not known, we can reference two groups of photoelectrons from two different targets to each other to determine the relative phase difference $\Delta \varphi$. The relative phase difference $\Delta \varphi$ is not sensitive to measurementinduced artifacts under the assumption that both groups of photoelectrons are equally influenced by (i) the continuum-continuum transition induced by the probing NIR field and (ii) the phase variation which inherently comes from the HHs generation (XUV chirp) [26]. In our experiments, we therefore performed a synchronous RABBITT measurement on $\mathrm{Ne}$ as the reference and $\mathrm{H}_{2}$ as the target gas, comparing SBs generated by the same couple of harmonics. In this way, we can cancel out the XUV-chirp term.

If we consider $\varphi_{(\mathrm{Ne}) \text { calc }}^{S_{2 q}}$ as known a priori, we can write

$$
\Delta \varphi_{\mathrm{H}^{+}}^{S_{2 q}}=\varphi_{\left(\mathrm{H}^{+}+\mathrm{Ne}^{+}\right) \exp }^{S_{2 q}}-\varphi_{\left(\mathrm{Ne}^{+}\right) \text {calc }}^{S_{2 q}}
$$

Figure 2(a) reports the extracted absolute EWP phases for the dissociative ionization of $\mathrm{H}_{2}$ from the attosecond streaking and the RABBITT measurements as a function of the photon energy. As stated, we select the $\mathrm{H}^{+}$fragments corresponding to the parallel configuration, in which the only optically allowed symmetries for the final states are $\Sigma_{u}$ (populated upon XUV one-photon absorption from the ground state) and $\Sigma_{g}$ (populated by the combination of an 

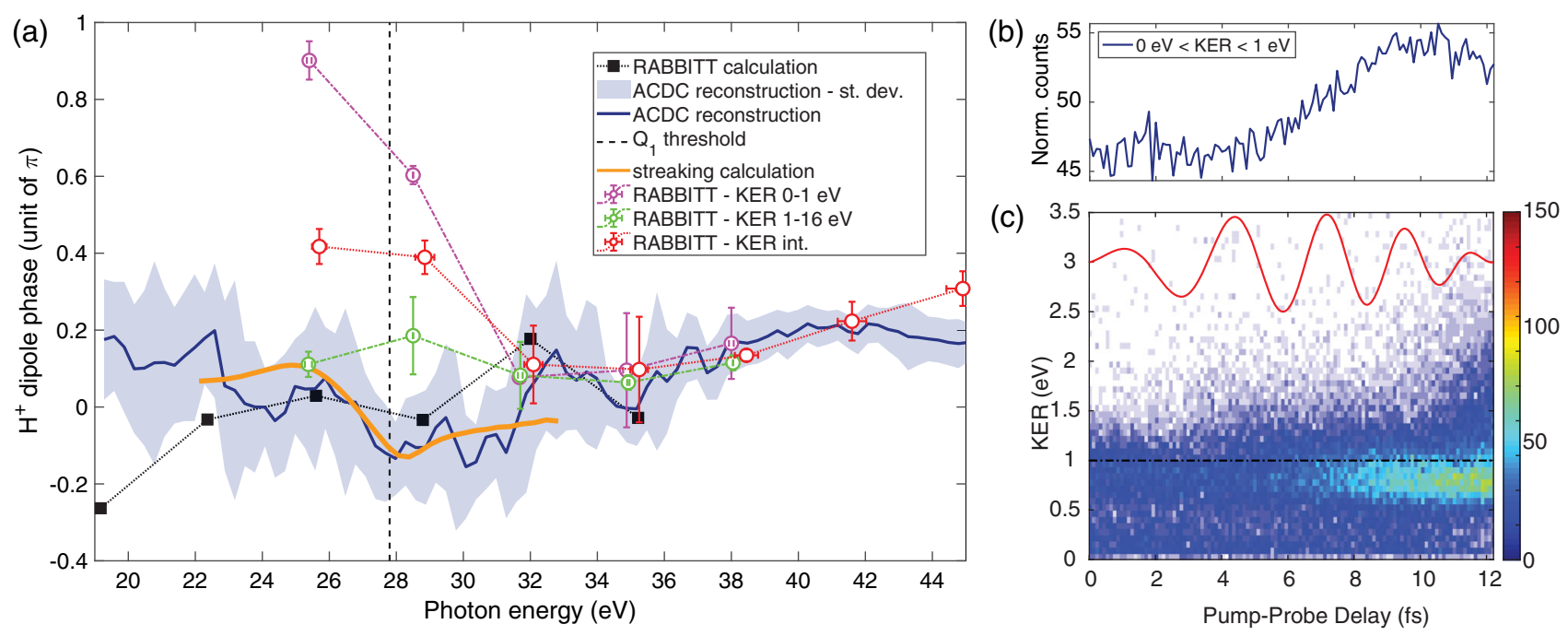

FIG. 2. (a) The blue curve and the blue shaded area show the experimental dipole phase and its standard deviation of the dissociative reaction of the $\mathrm{H}_{2}$ and $\beta$ parallel reconstructed using the ACDC algorithm. The red, magenta, and green symbols are the experimental RABBIT results for the KER integrated, $E_{k}<1 \mathrm{eV}$ and $E_{k}>1 \mathrm{eV}$ cases, respectively. The black dashed line indicates the threshold of $27.8 \mathrm{eV}$ above which the $Q_{1}$ DESs are populated. The parallel configuration corresponds to an angular selection of the photoemitted electrons of $\alpha=0 \pm 30^{\circ}$ and $\beta=0^{\circ}-0^{\circ}$ and $150^{\circ}-180^{\circ}$ for the streaking case, and $\alpha=0 \pm 30^{\circ} ; 180 \pm 30^{\circ}$ and $\beta=0^{\circ}-20^{\circ}$ and $160^{\circ}$ $-180^{\circ}$, for the RABBIT case. The black squares and the orange curve are the theoretical contributions for the RABBIT and streaking case, respectively. (b) and (c) Experimental KER as a function of the streaking pump-probe delay for the parallel orientation. The black dash-dotted line represents the $E_{k}=1 \mathrm{eV}$ while the red line shows the reconstructed IR streaking field from the Ne spectrogram. In detail (b) shows the integrated signal for $E_{k} \leq 1 \mathrm{eV}$ where a significant increase is observed after the $\sim 7 \mathrm{fs}$ pump-probe delay.

XUV and an NIR photon). A vertical transition from the ground state reaches the lowest $Q_{1}$ DES of $\Sigma_{u}$ symmetry in an energy range between 25 and $33 \mathrm{eV}$, with a maximum in the Franck-Condon region at around $28 \mathrm{eV}$, as indicated with a dashed line in Fig. 2(a). Above $31 \mathrm{eV}$, the second $Q_{2}$ series of DESs is also energetically open.

The ACDC reconstruction of the experimental streaking spectrograms and its standard deviation are represented by the blue line and the blue shaded area. The RABBITT data points, on the other hand, are denoted by red circles with related error bars. Details about standard deviation and error bar definitions can be found in the Supplemental Material [16].

A very good agreement between the streaking curve and the RABBITT data points is observed only above $30 \mathrm{eV}$. On the contrary, for the data points between 25 and $30 \mathrm{eV}$, located around the $Q_{1}$ threshold, a deviation is clearly visible.

To explain such a behavior, we supported our experimental results with theoretical $a b$ initio calculations reproducing both experimental conditions for the RABBITT and attosecond streaking measurements. The phases have been retrieved from the calculated photoelectron spectra using the same numerical procedure as for the experimental one. We first compare the experimental results with $a b$ initio calculations for the streaking case. The calculations have been performed by solving the timedependent Schrödinger equation with an NIR pulse of peak intensity $3 \times 10^{11} \mathrm{~W} / \mathrm{cm}^{2}$ and a duration of $6 \mathrm{fs}$, and an XUV pulse with peak intensity of $10^{9} \mathrm{~W} / \mathrm{cm}^{2}$, a duration of $1 \mathrm{fs}$ and a center energy of $28 \mathrm{eV}$. The choice of these parameters allows for a fine energy scan around $28 \mathrm{eV}$ where the lowest $Q_{1}$ resonance is populated. Details about the $a b$ initio calculations can be found in [11]. From the reconstruction of the calculated streaking spectrogram using the ACDC algorithm we obtained the orange curve shown in Fig. 2(a), where a remarkably good agreement is observed with the experimental streaking result. In particular, both the experimental and theoretical results highlight a dip in the dipole phase at the photon energy of $\sim 28 \mathrm{eV}$ where the lowest $Q_{1}$ doubly excited states of $\mathrm{H}_{2}$ are significantly populated.

The theoretical RABBITT results are shown in Fig. 2 (black squares). They have been obtained with an NIR pulse with peak intensity of $3 \times 10^{11} \mathrm{~W} / \mathrm{cm}^{2}$ and a duration of only 7.8 fs (as in Ref. [11]), which is much shorter than in the RABBIT experiments $\left(Q_{1}\right)$, thus leading to a substantial reduction of computer time. As can be observed, the theoretical RABBITT data points are in better agreement with the experimental streaking curves than they are with the experimental RABBITT results. This observation can be explained by the bond softening [27-29] induced by the relatively long ( $\sim 25-30 \mathrm{fs})$ NIR pulse used in the RABBITT measurements, which is not described by the theoretical model with an NIR pulse of only $7.8 \mathrm{fs}$. Bond softening effects associated to the dissociation of $\mathrm{H}_{2}$ 
start to appear for NIR pulses longer than 10 fs [15]. Such time is required for the NWP to reach larger internuclear distances where the coupling between the two involved ionic states, $1 s \sigma_{g}$ and $2 p \sigma_{u}$, is strong enough. This explains the good agreement between the calculated RABBITT data points and the result from the reconstruction of the experimental streaking spectrogram $(\sim 7.8 \mathrm{fs})$. Even at moderate NIR intensities of $I_{0}=1.4 \times 10^{11} \mathrm{~W} / \mathrm{cm}^{2}$, as in our RABBITT experiment (see Supplemental Material [16]), the NIR field plays an active role in the evolution of the molecule toward the dissociation limit [30].

In particular, the NIR field can dress the potential energy surfaces allowing for alternative fragmentation channels for certain vibrational states. This results in ionic fragments with slightly higher energy around $1 \mathrm{eV}$ (see Supplemental Material [16]). By selecting ionic products with $E_{k}>1 \mathrm{eV}$, we can reduce the contribution of the bond softening effect and approach the "ideal" perturbative regime in which the RABBITT technique is supposed to work [1,2,31]. As can be seen in Fig. 2(a) (green data points), the experimental RABBITT delays obtained from ions with $E_{k}>1 \mathrm{eV}$ are in better agreement with the experimental and theoretical streaking delays. On the other hand, the deviation becomes more pronounced if only ionic products with $E_{k}<1 \mathrm{eV}$ are considered (magenta data points), since this is the region where bond softening mainly manifests.

The results above $30 \mathrm{eV}$ in photon energy are not affected by the KER selection since for increasing photon energies, the KER also increases, reducing the relative importance of bond softening. Nevertheless, bond softening, though having a minor effect on the measured streaking patterns, cannot be completely ruled out. Figures 2(b) and 2(c) illustrate this point by showing the evolution of the KER in the region $0-3.5 \mathrm{eV}$ as a function of the pump-probe delay. The increase of the $\left(\mathrm{H}^{+}+\mathrm{H}\right)$ yield for $E_{k}<1 \mathrm{eV}$ occurring at pump-probe delays $\geq 7 \mathrm{fs}$ is the signature of the bond softening effect, although its relative contribution is not significant enough to distort the actual ionization delays. Similar effects have been reported from calculation on $\mathrm{H}^{+}[10,15]$ and from experiment on $\mathrm{D}^{+}[15]$. Note in contrast with the above findings, for the perpendicular molecular orientation, ionization delays obtained from our RABBITT and streaking measurements are in very good agreement with each other (see Supplemental Material [16]), since, as is well known [23], the coupling between the $1 s \sigma_{g}$ and $2 p \sigma_{u}$ states of the residual molecular cation induced by the NIR field at long pump-probe delays is negligible, thus preventing the appearance of bond softening.

In summary, we have retrieved ionization time delays in the hydrogen molecule from RABBITT and streaking measurements by using the ACDC algorithm. In combination with a COLTRIMS apparatus, we have disentangled the different ionization processes by selecting only specific ion products, molecular orientation and KER. For molecules parallel to the polarization direction of the XUV and IR pulses, RABBITT and streaking results strongly disagree for photon energies below $30 \mathrm{eV}$. We have demonstrated that, even at relatively low intensity of the IR probe pulse, the RABBITT experimental data are very sensitive to bond softening while streaking results are relatively unaffected due to the shorter IR pulse durations used in these kinds of measurements. The reconstruction of the streaking measurements using the ACDC algorithm shows a very good agreement with the ab initio calculations. As the use of long IR pulses is a necessary condition for the RABBITT technique to work, bond softening effects are likely to affect the retrieved phase delays in many molecules containing fast-moving nuclei. On the other hand, streaking methods, when properly analyzed, seem to be more appropriate to determine electron ionization delays in these targets due to the use of much shorter streaking waveforms and the ability to retrieve continuous phase and time-delay profiles in a single measurement.

This work was supported by NCCR Molecular Ultrafast Science and Technology (NCCR MUST), a research instrument of the Swiss National Science Foundation (SNSF) and by the European COST Action AttoChem. P. D. K. acknowledges support by the Air Force Office of Scientific Research (AFOSR) Grant under Contract No. FA9550-19-1-0065. For the Madrid team: all calculations were performed at the Mare Nostrum Supercomputer of the Red Española de Supercomputación (BSC-RES) and the Centro de Computación Científica de la Universidad Autónoma de Madrid (CCC-UAM). Work partially supported by the Spanish MICINN projects PID2019-105458RB-I00, the "Severo Ochoa" Programme for Centres of Excellence in R\&D (SEV-2016-0686) and the "María de Maeztu" Programme for Units of Excellence in R\&D (CEX2018000805-M), and the Comunidad de Madrid Synergy Grant FULMATEN.

*Present address: Max-Planck-Institut für Kernphysik, Saupfercheckweg 1, 69117, Heidelberg, Germany. These authors contributed equally to this work.

[1] H. G. Muller, Reconstruction of attosecond harmonic beating by interference of two-photon transitions, Appl. Phys. B 74, s17 (2002).

[2] P. M. Paul, E. S. Toma, P. Breger, G. Mullot, F. Augé, P. Balcou, H. G. Muller, and P. Agostini, Observation of a train of attosecond pulses from high harmonic generation, Science 292, 1689 (2001).

[3] P. Eckle, M. Smolarski, P. Schlup, J. Biegert, A. Staudte, M. Schöffler, H. G. Muller, R. Dörner, and U. Keller, Attosecond angular streaking, Nat. Phys. 4, 565 (2008).

[4] J. Itatani, F. Quéré, G. L. Yudin, M. Y. Ivanov, F. Krausz, and P. B. Corkum, Attosecond Streak Camera, Phys. Rev. Lett. 88, 173903 (2002). 
[5] V. Gruson, L. Barreau, Á. Jiménez-Galan, F. Risoud, J. Caillat, A. Maquet, B. Carré, F. Lepetit, J. F. Hergott, T. Ruchon, L. Argenti, R. Taïeb, F. Martín, and P. Salières, Attosecond dynamics through a Fano resonance: Monitoring the birth of a photoelectron, Science 354, 734 (2016).

[6] D. Azoury, O. Kneller, S. Rozen, B. D. Bruner, A. Clergerie, Y. Mairesse, B. Fabre, B. Pons, N. Dudovich, and M. Krüger, Electronic wavefunctions probed by all-optical attosecond interferometry, Nat. Photonics 13, 54 (2019).

[7] M. Kotur, D. Guénot, Á. Jiménez-Galán, D. Kroon, E. W. Larsen, M. Louisy, S. Bengtsson, M. Miranda, J. Mauritsson, C. L. Arnold, S. E. Canton, M. Gisselbrecht, T. Carette, J. M. Dahlström, E. Lindroth, A. Maquet, L. Argenti, F. Martín, and A. L'Huillier, Spectral phase measurement of a Fano resonance using tunable attosecond pulses, Nat. Commun. 7, 10566 (2016).

[8] J. Fuchs, N. Douguet, S. Donsa, F. Martín, J. Burgdörfer, L. Argenti, L. Cattaneo, and U. Keller, Towards the complete phase profiling of attosecond wave packets, Phys. Rev. Research 3, 013195 (2021).

[9] J. Fuchs, N. Douguet, S. Donsa, F. Martin, J. Burgdörfer, L. Argenti, L. Cattaneo, and U. Keller, Time delays from onephoton transitions in the continuum, Optica 7, 154 (2020).

[10] A. Palacios, A. González-Castrillo, and F. Martín, Electron streaking and dissociation in laser-assisted photoionization of molecular hydrogen, J. Phys. B 47, 124013 (2014).

[11] L. Cattaneo, J. Vos, R. Y. Bello, A. Palacios, S. Heuser, L. Pedrelli, M. Lucchini, C. Cirelli, F. Martín, and U. Keller, Attosecond coupled electron and nuclear dynamics in dissociative ionization of $\mathrm{H}_{2}$, Nat. Phys. 14, 733 (2018).

[12] L. Cattaneo, J. Vos, M. Lucchini, L. Gallmann, C. Cirelli, and U. Keller, Comparison of attosecond streaking and RABBITT, Opt. Express 24, 29060 (2016).

[13] H. Wei, T. Morishita, and C. D. Lin, Critical evaluation of attosecond time delays retrieved from photoelectron streaking measurements, Phys. Rev. A 93, 053412 (2016).

[14] X. Zhao, H. Wei, C. Wei, and C. D. Lin, A new method for accurate retrieval of atomic dipole phase or photoionization group delay in attosecond photoelectron streaking experiments, J. Opt. (United Kingdom) 19, 114009 (2017).

[15] G. Sansone, F. Kelkensberg, J. F. Pérez-Torres, F. Morales, M. F. Kling, W. Siu, O. Ghafur, P. Johnsson, M. Swoboda, E. Benedetti, F. Ferrari, F. Lépine, J. L. Sanz-Vicario, S. Zherebtsov, I. Znakovskaya, A. Lhuillier, M. Y. Ivanov, M. Nisoli, F. Martín, and M. J. J. Vrakking, Electron localization following attosecond molecular photoionization, Nature (London) 465, 763 (2010).

[16] See Supplemental Material at http://link.aps.org/supplemental/ 10.1103/PhysRevLett.128.063001 for more information about experimental RABBITT traces and analysis, streaking spectrograms, time-of-flight analysis, peak intensity estimation, reconstructued dipole phase of $\mathrm{H}^{2+}$ and $\mathrm{H}^{+}$using both RABBITT and streaking techniques, and the theoretical calculations presented in the manuscript.
[17] R. Dörner, V. Mergel, O. Jagutzki, L. Spielberger, J. Ullrich, R. Moshammer, and H. Schmidt-Böcking, Cold target recoil ion momentum spectroscopy: A "momentum microscope" to view atomic collision dynamics, Phys. Rep. 330, 95 (2000).

[18] M. Sabbar, S. Heuser, R. Boge, M. Lucchini, L. Gallmann, C. Cirelli, and U. Keller, Combining attosecond XUV pulses with coincidence spectroscopy, Rev. Sci. Instrum. 85, 103113 (2014).

[19] R. N. Zare, Dissociation of $\mathrm{H}_{2}^{+}$by electron impact: Calculated angular distribution, J. Chem. Phys. 47, 204 (1967).

[20] R. N. Zare, Photoejection dynamics, Mol. Photochem. 4, 1 (1972).

[21] A. Palacios, J. L. Sanz-Vicario, and F. Martín, Theoretical methods for attosecond electron and nuclear dynamics: Applications to the $\mathrm{H}_{2}$ molecule, J. Phys. B 48, 242001 (2015).

[22] S. Bhardwaj, S. K. Son, K. H. Hong, C. J. Lai, F. X. Kärtner, and R. Santra, Recombination-amplitude calculations of noble gases, in both length and acceleration forms, beyond the strong-field approximation, Phys. Rev. A 88, 053405 (2013).

[23] P. D. Keathley, S. Bhardwaj, J. Moses, G. Laurent, and F. X. Kärtner, Volkov transform generalized projection algorithm for attosecond pulse characterization, New J. Phys. 18, 073009 (2016).

[24] L. Pedrelli, P. D. Keathley, L. Cattaneo, F. X. Kärtner, and U. Keller, Complete phase retrieval of photoelectron wavepackets, New J. Phys. 22, 053028 (2020).

[25] E. P. Wigner, Lower limit for the energy derivative of the scattering phase shift, Phys. Rev. 98, 145 (1955).

[26] J. M. Dahlström, D. Guénot, K. Klünder, M. Gisselbrecht, J. Mauritsson, A. L'Huillier, A. Maquet, and R. Taïeb, Theory of attosecond delays in laser-assisted photoionization, Chem. Phys. 414, 53 (2013).

[27] P. H. Bucksbaum, A. Zavriyev, H. G. Muller, and D. W. Schumacher, Softening of the Molecular Bond in Intense Laser Fields, Phys. Rev. Lett. 64, 1883 (1990).

[28] H. G. Muller, P. H. Bucksbaum, A. Zavriyev, and D. W. Schumacher, Softening of the molecular bond in intense laser fields, AIP Conf. Proc. 233, 325 (2008).

[29] A. Zavriyev, P. H. Bucksbaum, H. G. Muller, and D. W. Schumacher, Ionization and dissociation of $\mathrm{H}_{2}$ in intense laser fields at $1.064 \mu \mathrm{m}, 532 \mathrm{~nm}$, and $355 \mathrm{~nm}$, Phys. Rev. A 42, 5500 (1990).

[30] M. Swoboda, J. M. Dahlström, T. Ruchon, P. Johnsson, J. Mauritsson, A. L'Huillier, and K. J. Schafer, Intensity dependence of laser-assisted attosecond photoionization spectra, Laser Phys. 19, 1591 (2009).

[31] V. Véniard, R. Taïeb, and A. Maquet, Phase dependence of $(N 1)$-color $(N>1)$ IR-UV photoionization of atoms with higher harmonics, Phys. Rev. A 54, 721 (1996). 